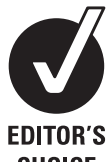

CHOICE

- An additional appendix is published online only. To view this file please visit the journal online (http://pmj.bmj.com/ content/88/1037.toc)

${ }^{1}$ Centre for Patient Safety and Service Quality, Department of Surgery and Cancer, Imperial

College London, UK

${ }^{2}$ Postgraduate Medical

Education Department, Central

Manchester University Hospitals

NHS Foundation Trust,

Manchester, UK

\section{Correspondence to}

Dr Maria Ahmed, Clinical

Research Fellow in Education and Training for Quality and

Safety, Centre for Patient Safety and Service Quality, Room 504

Medical School Building

Imperial College (St Mary's

Campus), Norfolk Place, London

W2 1PG, UK;

maria.ahmed@imperial.ac.uk

Received 4 July 2011

Accepted 5 December 2011

Published Online First

13 January 2012

\title{
Junior doctors' reflections on patient safety
}

\author{
Maria Ahmed, ${ }^{1}$ Sonal Arora, ${ }^{1}$ Simon Carley, ${ }^{2}$ Nick Sevdalis, ${ }^{1}$ Graham Neale ${ }^{1}$
}

\section{ABSTRACT}

Aim To determine whether foundation year 1 (FY1) doctors reflect upon patient safety incidents (PSIs) within their portfolios and the potential value of such reflections for quality of care.

Methods A cross-sectional retrospective review of every 'reflective practice' portfolio entry made by all FY1 doctors within an Acute Teaching Hospital Trust was conducted in February 2010. Entries were reviewed by two independent blinded researchers to determine whether they related to a PSI that is, any unintended or unexpected incident that could have or did lead to patient harm. For all entries rated positive by both reviewers, a content analysis approach was used to code PSI into incident type, contributing factors and patient outcome according to validated frameworks developed by the National Patient Safety Agency.

Results 139 reflective practice entries were completed by 30 trainees ( 15 men, 15 women, mean age 24 years). Of the 139 entries, $49 \%$ reflected on a PSI. Of these, $22 \%$ were due to errors in clinical assessment; $22 \%$ were due to delayed access to care; $18 \%$ were due to infrastructure/staffing deficiencies; and $16 \%$ were due to medication errors. The most common contributing factors were team/social factors (23\%), patient factors (22\%), communication and task factors (both 17\%). The majority of PSIs led to no harm. Six entries described PSIs resulting in patient death, the majority of which were attributable to diagnostic errors.

Conclusions FY1 doctors commonly reflect on PSIs within their professional portfolios. Such critical reflection can encourage learning but may also promote patient safety and the quality of healthcare across all medical specialties.

\section{INTRODUCTION}

Adverse events in the provision of healthcare continue to be a serious problem despite significant safety improvement efforts over the past decade. ${ }^{1}$ It is argued that substantive improvements in patient safety will be difficult to achieve without major reform in medical education at both undergraduate and postgraduate levels. ${ }^{1} 2$

As frontline clinicians, junior doctors are vital for promoting patient safety in the workplace. They will often be directly or indirectly involved in patient safety incidents (PSIs) and need to be encouraged to discuss and report such events in order to learn from them. ${ }^{3-6}$ However, evidence suggests that although 1 in 10 of hospitalised patients suffer from a PSI, relatively few such events are reported. ${ }^{7}$ Junior doctors may be particularly discouraged from reporting due to the perceived impact on their careers and the existence of a 'blame culture' rather than an 'open and learning' safety culture. ${ }^{8}$ Other methods are therefore required to identify PSIs, which junior doctors working at the bedside are aware of and likely to use to disclose such incidents.

We propose that reflection on clinical practice, which all doctors are strongly encouraged to perform, ${ }^{9}$ may be a fruitful way forward. Reflection refers to "those intellectual and affective activities in which individuals engage to explore their experiences in order to lead to a new understanding and appreciation." ${ }^{10}$ It is a powerful, metacognitive process that can lead to a greater understanding of the self and the situation to inform future actions. ${ }^{11}$ Reflection is rapidly becoming a competency in its own right and is being promoted in medical education as a core professional quality. ${ }^{9}$ Indeed, with the advent of revalidation, reflection on practice is one way in which licensed doctors in the UK will be expected to demonstrate continuous professional development and ultimately their fitness to practice. $^{12} \mathrm{~A}$ variety of educational approaches are commonly employed to facilitate reflective learning-including portfolios, reflective learning logs, multimedia approaches and models based on significant event analysis. ${ }^{11} 13$

Of these methods, portfolios in particular are being used increasingly throughout continuing medical education to encourage reflection, enhance personal responsibility for learning and support professional development. ${ }^{14} 15$ Within the UK, all trainees within the foundation programme (twoyear training programme forming the bridge between medical school and specialist training-see box 1) are now required to maintain an electronic portfolio of professional development and reflective learning competencies. ${ }^{16}$ Although patient safety awareness is recognised as a core element of medical professionalism, ${ }^{17-19}$ to the best of our knowledge no study to date has explored whether these portfolios can be used to assess awareness of patient safety issues. The aim of the study reported here was to evaluate whether foundation year 1 (FY1) doctors reflect on PSIs within their portfolios and to explore the potential value of such reflections for quality of care.

\section{METHODS \\ Study design}

This was a cross-sectional, retrospective record review of educational portfolios.

\section{Study participants}

Participants included all FY1 doctors $(n=30)$ in a University Hospital Foundation Trust. As a first study to explore the reflective practice of doctors on patient safety, we felt it appropriate to evaluate the practice of FY1 doctors for the following reasons:

- The foundation programme is a well-defined programme of clinical training within UK 


\section{Box 1 The foundation programme curriculum aims ${ }^{16}$}

The foundation programme curriculum provides the educational framework to support the professional development of new doctors in the first 2 years of clinical practice. The curriculum is based on the General Medical Council's 'Good Medical Practice' document which provides guidance on the principles and standards of clinical care, competence and conduct. ${ }^{17}$

Foundation Year 1: Aims to build upon the competences acquired during undergraduate training, completion of which leads to full registration with the General Medical Council (regulatory body for UK doctors).

Foundation Year 2: Aims to build upon Foundation Year 1 with a specific focus on the assessment and management of the acutely ill patient as well as generic professional skills (teamworking, communication, time-management).

postgraduate medical education with clear requirements regarding reflective practice.

- The foundation curriculum stipulates the central theme of patient safety within its contents so it would be apt to evaluate the extent to which foundation trainees (specifically FY1 doctors) appreciate this.

- Foundation programmes are locally managed with clear lines of accountability providing relative ease of access to portfolios for research purposes.

As all doctors were included from the entire year group in the study, no sampling was required. Consent to obtain 'reflective Practice' portfolio entries was obtained from the site foundation programme director. All 'reflective practice' entries made by FY1 doctors in their first year of clinical practice (from July 2008 to August 2009) were extracted by the foundation programme director from the e-portfolio provider ('Horus'). Entries were issued to the reviewers in an anonymised form so as to maintain confidentiality. Any data which may have identified any individual doctor or patient was screened out by the foundation programme director. The local research ethics committee confirmed that formal ethical approval was not required for this review of anonymised data.

\section{Procedure}

'Reflective practice' entries were typically structured around a proforma comprising the points below. Eleven of the 139 entries (8\%) were written in prose form but still addressed the recommended points.

1. Describe interesting, difficult or uncomfortable experiences.

Try to record both positive and not so positive elements.

What made the experience memorable?

2. How did it affect you?

3. How did it affect the team?

4. What did you learn from the experience and what (if anything) would you do differently next time?

Entries were reviewed retrospectively by independent and blinded reviewers with a medical background (MA, GN) to determine whether they related to a PSI-that is, "any unintended or unexpected incident that could have or did lead to harm for one or more persons receiving NHS-funded healthcare." ${ }^{20}$ Both reviewers had been previously trained and calibrated to perform the portfolio reviews. MA served as primary coder (ie, read each entry and applied the coding framework to it - see below) and any disagreements were settled through discussion/consensus with a third reviewer (SA).
Rather than develop a bespoke coding framework, the reviewers sought to analyse the portfolios using an existing PSI taxonomy. The UK-based National Patient Safety Agency guide 'Seven Steps to Patient Safety' was chosen for this purpose. ${ }^{20}$ This report describes the steps that NHS organisations need to take to improve safety and highlights the recommended dataset domains and taxonomy to be used for voluntary reporting of PSIs at national level. This national taxonomy was used to derive the proforma for the description and analysis of the reflective practice entries used by the reviewers in this study (see online appendix 1).

\section{Data analysis}

All portfolio entries were first read and re-read to ensure full immersion in the data. For all entries rated positive (ie, PSI identified) a content analysis approach was used to code the PSI into incident type, contributing factors and patient outcome using the proforma (online appendix 1). It was also noted whether the PSI was formally reported through the Hospital Incident Reporting Systems (HIRS) (through trainee self-report within the reflective entry).

The level of coding agreement between the two reviewers was assessed using Cohen's $\kappa$ coefficient. Descriptive statistics using SPSS V.17.0 were used to calculate absolute and relative (\%) frequencies of PSIs, their type, contributing factors and outcomes. CIs $(95 \%)$ are reported with all statistical indices.

\section{RESULTS}

One hundred and thirty-nine reflective entries were completed by 30 trainees ( 15 men, 15 women; mean age 24 years). On average, each trainee made five reflective entries. Of the total number of these 139 entries, 68 (49\%, 95\% CI 41\% to 57\%) reflected on a PSI. The formal reporting of PSIs to HIRS was mentioned in only 3 ( $4 \%, 95 \%$ CI $0.7 \%$ to $6 \%$ ) of these cases.

\section{Inter-rater reliability}

Inter-rater reliability analyses revealed that the coding of the incidents was robust for presence of a PSI $(\kappa=0.83,95 \%$ CI 0.73 to 0.92 ), type of PSI ( $\kappa=0.82,95 \%$ CI 0.71 to 0.94 ) and patient outcome ( $\kappa=0.70,95 \%$ CI 0.56 to 0.85 ).

\section{Incident type}

Certain types of incidents were more commonly reflected on than others. Fifteen of the 68 PSIs $(22 \%, 95 \%$ CI $14 \%$ to $33 \%)$ were due to errors in clinical assessment (delayed or wrong diagnosis) (table 1). Examples of this type of incident extracted from the portfolios were as follows:

"A gentleman with a history of resected laryngeal cancer was admitted to the ward as 'off legs'. On our ward round he was found to have neurological signs of acute cord compression that had not been picked up previously." (FY1 doctor 8)

"The fact that I incorrectly diagnosed acute pulmonary oedema as an escalating pneumonia and consequently almost gave the patient further fluids to treat her hypotension was very frightening." (FY1 doctor 11)

A further $15(22 \%)$ of PSIs were due to delay or failure to access care. For example:

"I was shocked that despite communicating my senior-approved management plan to the staff on day duty that it had not been carried out - with the result that the patient received suboptimal care. His Trop $\mathrm{T}$ (cardiac troponin) was still positive 3 days later and he had not had any further clexane (low-molecular weight heparin) since attending A\&E." (FY1 doctor 30) 
Table 1 Type of patient safety incident reflected on

\begin{tabular}{ll}
\hline Incident type* & Frequency \\
\hline Access, admission, transfer & 15 \\
Clinical assessment & 15 \\
Infrastructure including staffing, facilities, environment & 12 \\
Medication & 11 \\
Disruptive, aggressive behaviour & 5 \\
Implementation of care and ongoing monitoring/review & 4 \\
Self-harming behaviour & 3 \\
Treatment, procedure & 2 \\
Consent, communication, confidentiality & 1 \\
Documentation including records, identification & 0 \\
Infection control incident & 0 \\
Medical device/equipment & 0 \\
Patient abuse (by staff/third party) & 0 \\
Patient accident & 0 \\
\hline
\end{tabular}

*Please refer to the proforma in online appendix 1 for further details regarding the categories

Twelve PSIs (18\%, 95\% CI 10\% to $28 \%$ ) were assigned to infrastructure/staffing deficiencies and 11 (16\%, 95\% CI 9\% to $27 \%)$ were assigned to medication errors. The remaining PSIs were due to disruptive or aggressive behaviour (7\%), failure to monitor/review (6\%), self-harming behaviour (4\%), treatment or procedure complication (3\%) and failure to communicate outside of team (1\%).

\section{Contributing factors}

Each PSI was associated with an average of 2 (range 1-4) contributing factors. Certain types of contributing factors were more commonly reflected upon than others (table 2). The most significantly cited contributing factors to PSIs were team and social factors $(23 \%, 95 \%$ CI $17 \%$ to $31 \%)$ and patient factors (22\%, $95 \%$ CI $16 \%$ to $29 \%)$. An example relating to both these factors is cited below:

"An 82-year-old woman was admitted to the surgical ward and developed a deep venous thrombosis. We commenced the patient on warfarin therapy as recommended by guidelines on the prescription sheet. The patient's International Normalised Ratio post loading was 8.1. The consultant in charge of the patient was extremely annoyed. I felt disheartened at being blamed for the situation and for potentially putting the patient at increased risk of bleeding. I learnt from this experience that each patient must be treated individually. The patient was frail and very small and doses prescribed generally for average sized patients may not be applicable to all patients." (FY1 doctor 22)

Task factors and communication factors were the next most common cause (both $18 \%$, 95\% CI $9 \%$ to $20 \%$ ) An example relating to both these factors is cited below:

Table 2 Contributing factors reflected on

\begin{tabular}{ll}
\hline Contributing factor* & Frequency \\
\hline Team and social factors & 31 \\
Patient factors & 29 \\
Task factors & 18 \\
Communication factors & 18 \\
Equipment and resources factors & 17 \\
Education and training factors & 10 \\
Working conditions and environmental & 6 \\
Individual factors & 5 \\
\hline
\end{tabular}

*Please refer to the proforma in online appendix 1 for further details regarding the categories.
"Phlebotomists provide a blood-taking service on the ward 3 times a week. On many occasions I would find that not all the bloods had been taken....I recommended that any bloods that cannot be done need to be brought to the attention of the junior doctors so that they can be attempted later. I realised from this experience that effective communication between members of healthcare staff is essential to provide patients with good medical care." (FY1 doctor 13)

\section{Outcome}

There was insufficient information to determine PSI outcome in $19(28 \%)$ cases. Of the entries where elucidation of outcome was possible $(n=49)$, the majority $(47 \%, 95 \%$ CI $34 \%$ to $61 \%)$ led to no harm to the patient. Six $(12 \%, 95 \%$ CI $6 \%$ to $24 \%)$ reflective entries described PSIs resulting in patient death (figure 1). The details of these deaths are presented in table 3.

\section{DISCUSSION}

Patient safety is a key item on the healthcare agenda, both nationally and internationally. ${ }^{21}$ Junior doctors are considered powerful 'agents for change' in promoting safety improvement in the workplace. ${ }^{22}$ This study shows that FY1 doctors commonly reflect on PSIs within their professional development portfolios-thus demonstrating awareness of patient safety issues in their everyday clinical practice. As new doctors embarking on lifelong careers within the UK healthcare system, this is reassuring especially in light of proposals for revalidation which will expect all licensed doctors to continually reflect and improve upon their practice. ${ }^{12}$

Although the reflective practice proforma does not specifically enquire into PSIs, nearly half of all reflective entries made by FY1 doctors described a PSI as a memorable event. This supports findings from other studies in the UK and abroad. ${ }^{4}{ }^{23}$ In one study, when young interns were asked "What were your most memorable experiences during training?", 21\% of the responses related to actual or potential mistakes. ${ }^{23}$ Within this study, errors in diagnosis, delay or failure to access care, deficiencies in infrastructure/staffing and medication errors were the most common types of PSI reflected on. These findings broadly mirror those found in a study which asked house officers to describe their most significant medical mistake in the last year. ${ }^{4}$ However, this pattern of incident type differs from that found in the data summaries from the UK's National Reporting and Learning System (NRLS). ${ }^{24}$ The latest national data shows that the most common PSIs reported via HIRS were patient accidents $(30 \%)$, followed by medication errors $(11 \%)$, treatment/procedural complications $(10 \%)$, delays or failure to access care $(8 \%)$ and errors in documentation and deficiencies in implementation

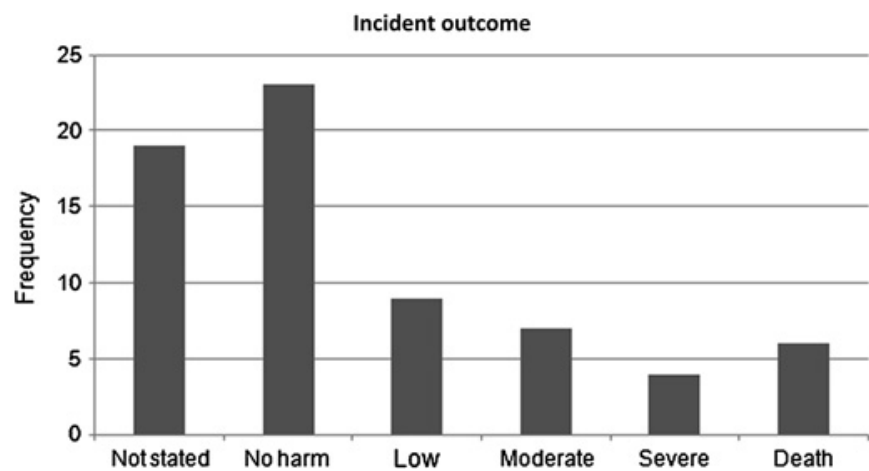

Figure 1 Outcome of patient safety incidents. 
Table 3 Detailed analysis of patient safety incidents with outcome 'severe harm/death'

\begin{tabular}{|c|c|c|}
\hline Incident type & Description & Outcome \\
\hline $\begin{array}{l}\text { Consent, communication: failure to } \\
\text { communicate outside of team }\end{array}$ & $\begin{array}{l}\text { Antibiotic treatment for methicillin-resistant Staphylococcus aureus septicaemia } \\
\text { incorrectly stopped due to failure to handover to new team }\end{array}$ & Death \\
\hline Clinical assessment: missed diagnosis & Missed diagnosis of spinal cord compression leading to paralysis and death & Death \\
\hline Clinical assessment: missed diagnosis & Delayed recognition and treatment of post-operative hypotension & Death \\
\hline Clinical assessment: missed diagnosis & Delayed diagnosis of ruptured abdominal aortic aneurysm & Death \\
\hline Treatment, procedure & Complication of abdominal aortic aneurysm repair (aorto-enteric fistula) & Death \\
\hline Clinical assessment: missed diagnosis & Missed diagnosis of acute coronary syndrome & Death \\
\hline Medication error & Medical cardioversion attempted in non-specialist setting, involving cardiac arrest & Severe harm \\
\hline Clinical assessment: missed diagnosis & Missed diagnosis of small bowel obstruction, leading to bowel resection & Severe harm \\
\hline Infrastructure, staffing & Emergency abdominal aortic aneurysm repair involving inexperienced theatre staff & Severe harm \\
\hline Infrastructure, staffing & Inappropriate transfer of complex case to ward setting (thoraco-abdominal aneurysm) & Severe harm \\
\hline
\end{tabular}

of ongoing care/monitoring (both 6\%). Errors in clinical assessment/diagnosis formed a minority of reported incidents at just $5 \%$. ${ }^{24}$ The different distribution of PSI type between national findings and reflections from our study probably reflects the different demographics of staff that report PSIs through HIRS. Nurses and allied health professionals tend to report incidents much more frequently than doctors. ${ }^{25}$ Hence diagnostic errors, most commonly reflected on by doctors, do not feature highly on national dataset summaries, whereas patient accidents involving slips, trips and falls reported most commonly by nursing staff feature heavily in such reports. ${ }^{25} 26$

Multiple factors were found to contribute to the PSIs reflected upon, supporting existing evidence that diverse factors spanning individual healthcare professionals and healthcare systems impact on patient safety. ${ }^{7}$ Data on contributing factors do not appear to be collated on a national scale, possibly in part reflecting the complexities involved in standardising and analysing such data. ${ }^{26}$ This suggests that reflective entries could be a useful source of information regarding factors that contribute to error and harm in a way currently difficult to achieve via national reporting systems. Moreover, the majority of PSIs led to no harm and this supports national data. ${ }^{24}$ Six PSIs led to patient death and on closer analysis, the majority of these incident types were attributable to errors in clinical assessment: missed or delayed diagnosis. This supports evidence to show that diagnosis-related incidents are more likely to be associated with moderate/severe harm/death than incidents unrelated to diagnosis. $^{26}$

\section{Limitations}

With regards to limitations, this study was conducted on a total cohort of FY1 doctors, thus avoiding selection bias. However, this study may not represent the reflective practice of junior doctors on a national scale and a larger study to include trainees working at different hospitals across the UK and internationally would be required to assess generalisability. The quality and depth of reflections made by trainees varied and as a result it was difficult to extract sufficient information from some entries. Entries varied from four to five sentences to up to a full page. Furthermore the incident outcome could not be elucidated in $28 \%$ of PSIs reflected on. Such variability in PSI reporting is not new-we have documented this in PSIs reported by experienced NHS clinicians. ${ }^{26}$ However, the high inter-rater reliability of the coding using a national taxonomy system suggests that such a framework would be useful at a national scale to identify PSIs from other reflective entries.

\section{Implications and further research}

These findings carry a number of implications. PSIs are a source of considerable emotional distress for junior doctors and if there is a 'blame culture' the clinician may form the second victim. $^{8} 27$ While only three of the PSIs reflected on were formally reported, it is clear that trainees are internalising such events and reflecting on them. Educational strategies should be implemented to encourage formal reporting and promote shared learning from PSIs. It is also possible that with an evident reluctance of physicians to report errors formally ${ }^{25}$ reflective portfolios may provide a novel data source to capture the trueprevalence of PSIs thereby enhancing learning at organisational level. A potential study should first map out the routes that doctors take to reporting and reflecting on PSIs. Different stages of training as well as different specialties ought to be considered and included here. Once the reporting and reflection routes have been identified, their comparability ought to be addressed. If comparable, such entries could be analysed using the framework we used in the present study. However, if shown non-comparable, a different design in which doctors would be specifically asked to recall and reflect on a PSI that has occurred to them or has come to their attention would be more suitable. Different study designs would certainly have different advantages and disadvantages - which should be considered carefully.

With these in mind, we do feel strongly that such a study would be valuable in identifying how doctors reflect about PSIs, as well as the type of PSIs that occur to them and are most memorable-this information would assist in understanding PSIs in current clinical practice beyond what we know from reporting systems like the NRLS. Apathy towards reporting to the NRLS, nurse-led reporting, and the perceived lack of feedback from the system are some of its limitations, which have contributed to low reporting from doctors. Portfolio analysis, and analysis of speciality-specific incident reporting and analysis (including Confidential Reporting System in Surgery for surgeons and Safe Anaesthesia Liaison Group for anaesthetists, for instance) ${ }^{28} 29$ could help address this gap.

Finally, further research is also required to assess whether reflection on PSIs actually translates into safer clinical practice. Some recent research suggests that critical reflection on such 'disorienting dilemmas' can stimulate improvements in both individual and systems-based practice through transformative learning-systematic empirical investigation is required to elucidate this issue. ${ }^{30} 31$

\section{CONCLUSIONS}

This study shows FY1 doctors commonly reflect on PSIs within their portfolios and that reflective entries may be a useful tool to determine junior doctors' awareness and understanding of PSIs. Such critical reflection can encourage learning but may also 


\section{Main messages}

As frontline clinicians junior doctors are vital to promoting patient safety in the workplace; however, incident reporting among this cohort is low.

- Portfolios are widely used across undergraduate and postgraduate medical education to promote reflective learning.

- Foundation Year 1 doctors commonly reflect upon patient safety incidents within their professional development portfolios.

- Foundation doctor portfolios may prove an additional source of data to detect adverse events in healthcare.

\section{Current research questions}

- How does the reflective practice on patient safety of specialty/senior doctors compare with that of Foundation Year 1 doctors?

- How can we promote wider organisational learning through the individual reflections on patient safety incidents?

- To what extent does reflection on patient safety incidents translate to safer clinical practice?

promote patient safety and the quality of healthcare across all medical specialties.

Funding This study did not receive specific funding. MA, SA, NS and GN are members of the Imperial Centre for Patient Safety and Service Quality, which is funded by the UK's National Institute for Health Research.

Competing interests None.

Contributors All authors contributed to the design of the study, draft and approval of the final manuscript. MA, SA and GN analysed and interpreted the data.

Provenance and peer review Not commissioned; externally peer reviewed.

\section{REFERENCES}

1. House of Commons Health Committee. Patient Safety: Sixth report of session 2008-09. http://www.publications.parliament.uk/pa/cm200809/cmselect/cmhealth/ cmhealth.htm (accessed 27 Jan 2011).

2. Lucian Leape Institute Roundtable on Reforming Medical Education. Unmet Needs: Teaching Physicians to Provide Safe Patient Care. Boston, MA: National patient safety foundation, 2010.

3. Long S, Neale G, Vincent C. Practising safely in the foundation years. BMJ 2009;338:b1046.

4. Wu AW, Folkman S, McPhee SJ, et al. Do house officers learn from their mistakes? JAMA 1991;265:2089-94.

5. National Patient Safety Agency. Medical Error: What To Do If Things Go Wrong A Guide for Junior Doctors. 2010. http://www.nrls.npsa.nhs.uk/resources/? Entryld45=74246 (accessed 27 Jan 2011).
6. Robson J, de Wet C, Mckay J, et al. Do we know what foundation year doctors think about patient safety incident reporting? Development of a web based tool to assess attitude and knowledge. Postgrad Med J 2011. 87:750-6.

7. Vincent C. Patient Safety. 2nd edn. Chichester, UK: Wiley-Blackwell, 2010

8. Wu AW. Medical error: the second victim. BMJ 2000;320:726

9. Gans RO. Mentoring with a formative portfolio: a case for reflection as a separate competency role. Med Teach 2009;31:883-4.

10. Moon JA. A Handbook of Reflective and Experiential Learning: Theory and Practice. Abingdon: Routledge Falmer, 2004

11. Sandars J. The use of reflection in medical education: AMEE Guide No. 44. Med Teach 2009;31:685-95.

12. General Medical Council. Revalidation: a Statement of Intent. 2010. http://www gmc-uk.org/Revalidation A Statement of Intent October 2010 Final version

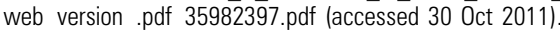

13. Grant AJ, Vermunt JD, Kinnersley $P$, et al. Exploring students' perceptions on the use of significant event analysis, as part of a portfolio assessment process in genera practice, as a tool for learning how to use reflection in learning. BMC Med Educ 2007:7:5

14. Tochel C, Haig A, Hesketh A. The effectiveness of portfolios for postgraduate assessment and education: BEME Guide No 12. Med Teach 2009;31:299-318.

15. Buckley S, Coleman J, Davison I, et al. The educational effects of portfolios on undergraduate student learning: a Best Evidence Medical Education (BEME) systematic review. BEME Guide No. 11. Med Teach 2009;31:282-98.

16. UK Foundation Programme Office. The UK Foundation Programme Curriculum http://www.foundationprogramme.nhs.uk/index.asp?page=home/keydocs\#c\&rg (accessed 20 May 2011).

17. General Medical Council. Good Medical Practice. 2009. http://www.gmc-uk.org/ static/documents/content/GMC GMP 0911.pdf (accessed 20 May 2010).

18. General Medical Council. Raising Concerns About Patient Safety. 2008. http:// www.gmc-uk.org/static/documents/content/Raising_concerns.pdf laccessed 20 May 2010).

19. General Medical Council. Tomorrow's Doctors. 2009. http://www.gmc-uk.org/ static/documents/content/TomorrowsDoctors_2009.pdf (accessed 20 May 2010)

20. National Patient Safety Agency. Seven Steps to Patient Safety: Full Reference Guide. 2004. http://www.nrls.npsa.nhs.uk/resources/collections/seven-steps-topatient-safety/?entryid45 $=59787$ (accessed 3 Jan 2010).

21. World Health Organisation. Conceptual Framework for the International Classification for Patient Safety: Final Technical Report. http://www.who.int/ patientsafety/taxonomy/icps_full_report.pdf (accessed 20 May 2012)

22. Junior doctors: agents for change. BMJ campaign. http://www.bmj.com/campaigns/ juniordoctors/ (accessed 20 May 2010)

23. Mizrahi T. Managing medical mistakes: ideology, insularity and accountability among internist-in-training. Soc Sci Med 1984:19:135-46.

24. National Patient Safety Agency. National Reporting and Learning System Quarterly Data Workbook up to December 2010, May 2011. http://www.nrls.npsa. nhs.uk/resources/collections/quarterly-data-summaries/?entryid45=131059 (accessed 1 Jul 2011).

25. Wallace LM, Koutantii M, Spurgeon P, et al. Reporting Systems: A Scoping Study of Methods of Providing Feedback within an Organization. Patient Safety Research Portfolio Report No 28. Coventry, UK: Coventry University, 2006.

26. Sevdalis N, Jacklin R, Arora S, et al. Diagnostic error in a national incident reporting system in the UK. J Eval Clin Pract 2010;16:1276-81.

27. Firth-Cozens J. Emotional distress in junior house officers. Br Med J (Clin Res Ed) 1987:295:533-6.

28. CORESS: A Confidential Reporting System for Surgery. http://www.coress.org.uk/ index.htmhttp://www.coress.org.uk/index.htm (accessed 30 0ct 2011).

29. SALG Safe Anaesthesia Liaison Group. http://www.rcoa.ac.uk/index.asp? PagelD=1512 (accessed 30 Oct 2011).

30. Wittich CM, Reed DA, McDonald FS et al. Transformative learning: a framework using critical reflection to link the improvement competencies in graduate medical education. Acad Med 2010;85:1790-3.

31. Cranton P. Understanding and Promoting Transformative Learning: a Guide for Educators of Adults. 2nd edn. San Francisco: Calif:Jossey-Bass, 2006 